\title{
Discrepant effects of mexiletine on cycle length of ventricular tachycardia and on the effective refractory period in the area of slow conduction
}

Yoshifusa Aizawa, Masaomi Chinushi, Hitoshi Kitazawa, Takashi Washizuka, Akira Abe, Akira Shibata, Itsuo Kodama

\begin{abstract}
Objective-Monomorphic sustained ventricular tachycardia (VT) can often be entrained and interrupted at a critical paced cycle length. The aim was to evaluate a possible determinant of this phenomenon by observing the action of mexiletine on the critical paced cycle length and other variables.
\end{abstract}

Methods-Nine consecutive patients with symptomatic VT were studied. After induction of VT, the area of slow conduction was mapped as the earliest site of the activation or the site with mid-diastolic potential during the tachycardia. Rapid pacing was performed at a site distant from the tachycardia circuit to entrain the tachycardia, starting at a cycle length 10-20 ms shorter than the VT cycle length, and repeated after a decrement of the cycle length in steps of $10 \mathrm{~ms}$ to obtain the longest paced cycle length that interrupted the tachycardia: the block cycle length. The effective refractory period (ERP) was measured at the pacing site at which the myocardium was presumed to be normal and also in the area of slow conduction. The effects of mexiletine on the cycle length of VT, the block cycle length, and the ERP at two sites were obtained before and after mexiletine administration. The relation between the cycle length of VT and block cycle length and their changes were also analysed.

Results-11 VTs with the same morphology were induced before and after mexiletine administration. The VT cycle length was prolonged by mexiletine from 309 (SD 53) to 361 (47) $\mathrm{ms}$, and it was interrupted at block cycle lengths of 247 (37) and $307(41) \mathrm{ms}$, respectively, the changes being 18 (12)\% and 23 (8)\% (both $P<$ $0 \cdot 001)$. All VTs were entrained, and during pacing at the block cycle length there was abrupt loss of fusion and change in the presystolic electrogram, always associated with interruption of VT on cessation of rapid pacing. A good correlation was observed between the VT cycle length and the block cycle length $(r=0.77$ to $0 \cdot 80$ ). The ERP at the pacing site (normal myocardium) and in the area of slow conduction showed no significant change: 241 (21) $v 240$ (22) ms and 262 (9) $v 252$ (9) ms, respectively. The block cycle length was longer than the ERP after mexiletine administration: 362 (55) v 252 (9) ms ( $\mathbf{P}<0.02)$.
Conclusions-Mexiletine prolonged the cycle length of VT and the VT-interrupting critical cycle length but not the ERP. The prolongation of the VT cycle length and the block cycle length by mexiletine seemed to be unrelated to the action potential duration, but related to depressed intercellular conduction.

(Heart 1996;75:281-286)

Keywords: mexiletine; ventricular tachycardia; cycle length; effective refractory period

Most episodes of sustained ventricular tachycardia (VT), including those unrelated to coronary artery disease, have been shown to be entrained with rapid ventricular pacing, ${ }^{12}$ indicating that the underlying mechanism is the presence of a reentrant circuit with an excitable gap. ${ }^{3-5}$

When transient entrainment with rapid ventricular pacing is repeated at progressively shorter cycle lengths, ventricular tachycardia is often interrupted at a critical cycle length, and we defined such a critical VT-interrupting cycle length as the block cycle length. ${ }^{126}$ During rapid pacing at the block cycle length, characteristic findings are observed in the presystolic electrogram and in the surface electrocardiogram ${ }^{126}$ and they are considered to be due to orthodromic block within the area of slow conduction. ${ }^{136}$

In our earlier study, ${ }^{6}$ procainamide was shown to prolong the block cycle length as well as the cycle length of ventricular tachycardia. Since procainamide prolongs the duration of the action potential by depressing the potassium channel, ${ }^{7}$ the prolongation of the block cycle could be due to the prolonged duration of the action potential.

Mexiletine is known to shorten the duration of the action potential, ${ }^{8-10}$ so we investigated its effects on the block cycle length in reentrant ventricular tachycardia in an attempt to clarify the determinants of the block cycle length. The effective refractory period measured in the area of slow conduction was compared with the block cycle length.

\section{Methods}

PATIENTS

Among 45 patients who underwent consecutive electrophysiological study for the selection of antiarrhythmic drugs, nine were chosen for the present study. All had symptomatic 
monomorphic sustained ventricular tachycardia which was inducible at a control study. The other inclusion criteria were as follows: (1) in a control study, rapid pacing was performed to entrain ventricular tachycardia at progressively shorter cycle length until the tachycardia was interrupted; (2) after administration of mexiletine, ventricular tachycardia with identical morphology to that of the control state was induced; (3) the reinduced ventricular tachycardia after mexiletine administration could be entrained and interrupted with rapid ventricular pacing at a critical cycle length. The criteria of transient entrainment of ventricular tachycardia with rapid ventricular pacing are described below.

The ages of the patients ranged from 16 to 77 years, with a mean of 55 (SD 23) years. Five were male and four were female. Five patients had ventricular tachycardia related to coronary artery disease and all had had myocardial infarction in the past (more than 10 months before). An occlusion of the coronary artery to the infarcted area was demonstrated in coronary angiograms. One female and one male patient had been operated on for double outlet right ventricle seven and 10 years previously. The remaining two patients had idiopathic cardiomyopathy. These latter four patients showed normal coronary arteriograms. Details of the patients are given in the table.

\section{ELECTROPHYSIOLOGICAL STUDY}

The procedure, purpose, and possible risks were fully explained. After informed and written consents were obtained, the electrophysiological study was performed in the postabsorptive and non-sedated state. All antiarrhythmic agents were discontinued for 2-3 d before the control study, and no patient had been treated with amiodarone.

Quadripolar electrode catheters (USCI, 6F multipurpose catheter) were placed against the right atrium, the $\mathrm{His}$ bundle region, and the apex or the outflow tract of the right ventricle. Another quadripolar electrode catheter was positioned within the left ventricle. Extensive endocardial catheter mapping of both ventricles was performed to determine the site of the earliest activation, ${ }^{11}$ or the site with a middiastolic electrogram during ventricular tachy-

Clinical and electrophysiological characteristics of ventricular tachycardia and the drug induced changes

\begin{tabular}{llllll} 
& & & & \multicolumn{2}{l}{$V T C L /$ block $C L($ ms $)$} \\
\cline { 5 - 6 } Patient & Age/sex & UDH & VT & Control & Mexiletine \\
\hline 1 & $72 \mathrm{M}$ & OMI & RB & $280 / 220$ & $300 / 280$ \\
2 & $72 \mathrm{M}$ & OMI & RB & $340 / 280$ & $410 / 340$ \\
3 & $70 \mathrm{M}$ & OMI & RB & $260 / 220$ & $320 / 300$ \\
4 & $67 \mathrm{M}$ & OMI & LB & $300 / 270$ & $340 / 310$ \\
5 & $77 \mathrm{~F}$ & OMI & RB & $260 / 200$ & $360 / 250$ \\
6 & $46 \mathrm{M}$ & DCM & RB & $370 / 320$ & $420 / 400$ \\
7 & $51 \mathrm{~F}$ & DCM & RB & $280 / 240$ & $370 / 320$ \\
8 & $16 \mathrm{M}$ & DORV & LB & $400 / 290$ & $420 / 340$ \\
9 & $21 \mathrm{~F}$ & DORV & LB & $260 / 240$ & $390 / 290$ \\
Mean (SD) & $55(23)$ & & & $309(53) / 247(37)$ & $361(45) / 307(41)$ \\
\hline
\end{tabular}

UDH, underlying heart disease; VT, ventricular tachycardia; CL, cycle length; Block CL, block cycle length; DCM, dilated cardiomyopathy; DORV, double outlet right ventricle; $\mathrm{LB}$, left bundle branch block-like morphology; OMI, old myocardial infarction; RB, right bundle branch block. cardia. In the latter site, criteria of concealed entrainment were fulfilled ${ }^{1213}$ and these sites were considered to be within the area of slow conduction closest to the exit from the central common pathway.

The standard protocol was used for the induction of ventricular tachycardia: the extrastimulus technique using one to two (three when necessary) extrastimuli after eight basic stimuli at two cycle lengths, $600 \mathrm{~ms}$ and $400 \mathrm{~ms}$; and incremental pacing at a cycle length between 600 and $286 \mathrm{~ms}$ for 5-15 s. ${ }^{126}$ Electrical stimuli of $2.0 \mathrm{~ms}$ duration were delivered at twice the late diastolic threshold by a programmable stimulator (Fukuda Denshi cardiac stimulator BCO2). The induction of ventricular tachycardia was attempted from the apex and the outflow tract of the right ventricle and from the left ventricle in that order.

The intracavitary electrograms were filtered at 30 to $500 \mathrm{~Hz}$ and stored on magnetic tape simultaneously with surface electrocardiographic leads I, II, and V1. They were retrieved later on a recorder. The data were also recorded directly on an ink jet recorder at a paper speed of $100 \mathrm{~mm} / \mathrm{s}$. During programmed stimulation, all induced arrhythmias were recorded on 12-lead electrocardiogram.

\section{RAPID VENTRICULAR PACING OF VENTRICULAR} TACHYCARDIA

After the induction of ventricular tachycardia, rapid pacing was performed from the apex of the right ventricle starting at a cycle length which was $10-20 \mathrm{~ms}$ shorter than that of ventricular tachycardia. When ventricular tachycardia was entrained, rapid pacing was repeated after a decrement of the paced cycle length in steps of $10 \mathrm{~ms}$ until the tachycardia was interrupted. ${ }^{126}$

When ventricular tachycardia was not entrained from the apex of the right ventricle, rapid pacing was performed from the outflow tract of the right ventricle. Rapid pacing from the left ventricle was used for patients in whom ventricular tachycardia could not be entrained from two sites of the right ventricle.

The first stimulus was given at $5 \mathrm{~ms}$ after triggering of the local electrogram at the pacing site so that the relation between the first stimulus, the local electrogram, and the QRS complex of the ventricular tachycardia was kept constant in each pacing.

Rapid pacing at each cycle length was continued for 5-15 s and the QRS complex and the presystolic electrograms were checked for the possible changes in the morphology and the timing of the activation. In our earlier studies, ${ }^{126}$ constant fusion was obtained following the initial four to six beats of pacing, and during pacing at the block cycle length characteristic changes in the presystolic electrogram and in the surface electrocardiogram were observed within $5 \mathrm{~s}$, invariably associated with interruption of ventricular tachycardia as evident on cessation of rapid pacing. Rapid pacing was performed at progressively shorter paced cycle lengths before and after mexiletine administration. 
A
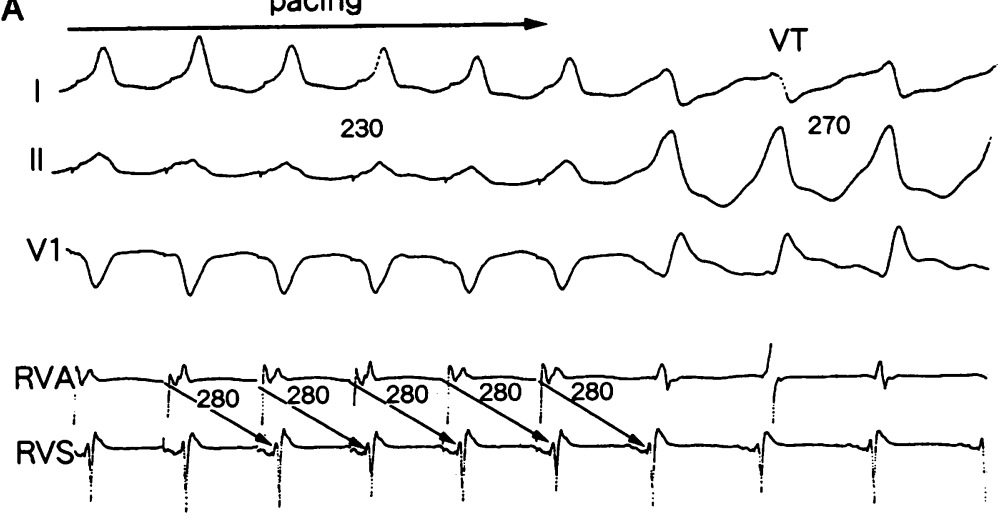

B

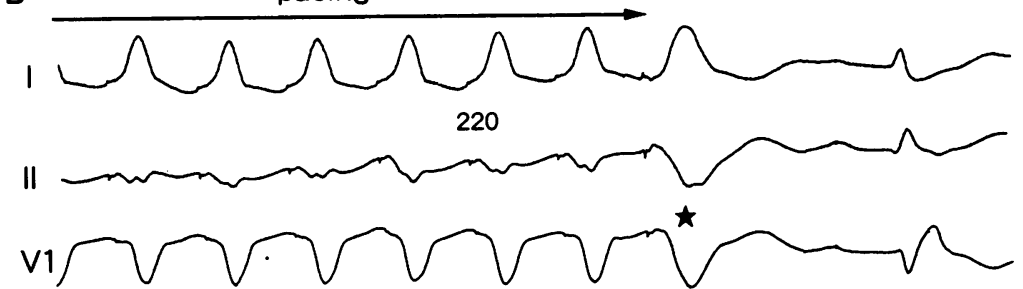

Figure 1 Rapid pacing and interruption of ventricular tachycardia (VT). The patient had VT after correction of double outlet right ventricle. The cycle length of the VT was $270 \mathrm{~ms}$ and rapid pacing was attempted at 250-220 ms from the apex of the right ventricle (RVA). (A) Rapid pacing at 230 ms shows constant fusion during pacing. The presystolic electrogram at RVS was activated orthodromically and the morphology was identical to that during VT. (B) During pacing at $220 \mathrm{~ms}$ constant fusion was followed by an abrupt loss of fusion, as shown by the asterisk. The local electrogram at RVS showed changes in the morphology and timing of the activation. For these changes, orthodromic block within the zone of slow conduction was considered to be responsible. The appearance of a single fully paced $Q R S$ complex occurring during pacing at the block cycle length was associated with interruption of VT. I, II, VI, surface leads; RVS, the site of ventricular tachycardia origin with presystolic local electrogram on the interventricular septum of the right ventricle.
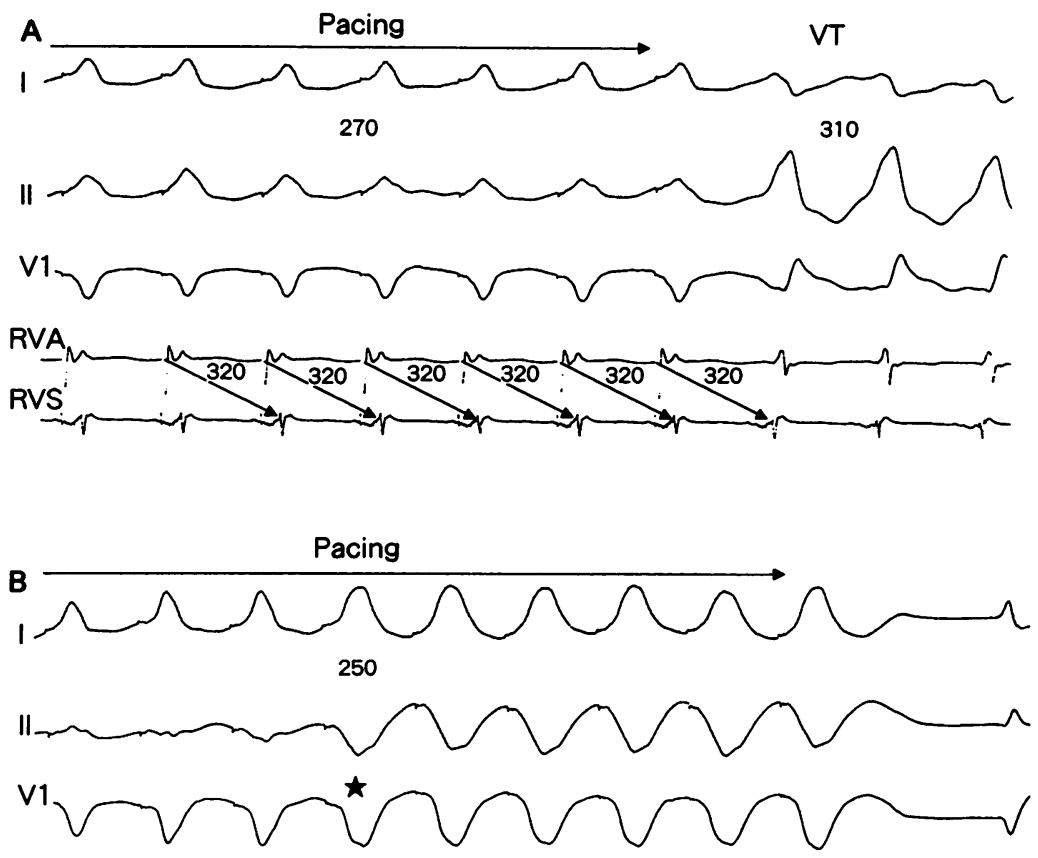

Figure 2 Transient entrainment and interruption of ventricular tachycardia. The patient was the same as in fig 1. After mexiletinl administration, the cycle length of ventricular tachycardia (VT) was prolonged to $310 \mathrm{~ms}(14 \cdot 8 \%)$. Rapid pacing was performed at 300-250 ms. Constant fusion and progressive fusion are obvious during pacing at two cycle lengths: $270 \mathrm{~ms}$ in $A$ and $250 \mathrm{~ms}$ in $B$, at which VT was interrupted. The block cycle length was prolonged from $220 \mathrm{~ms}$ to $250 \mathrm{~ms}$ by mexiletine. Abbreviations are same as in fig 1 .
CRITERIA OF TRANSIENT ENTRAINMENT

The criteria of transient entrainment by earlier workers $^{3}$ were used: (1) during ventricular tachycardia, while pacing at a constant rate that is faster than the rate of spontaneous ventricular tachycardia and which fails to interrupt it, the demonstration of constant fusion beats in the electrocardiogram, except for the last captured beat, which is not fused; (2) during ventricular tachycardia, while pacing at two or more constant rates that are faster than the rate of ventricular tachycardia but which fail to interrupt it, the demonstration of constant fusion beats on the electrocardiogram at each rate, but different degrees of constant fusion at each rate (progressive fusion); (3) during ventricular tachycardia, while pacing at a constant rate faster than the rate of ventricular tachycardia that interrupts the tachycardia, the demonstration of localised conduction block to the site for one beat followed by activation of the site by the next paced beat from a different direction and with a shorter conduction time.

When mid-diastolic electrical activity was recorded, rapid pacing was performed at the site to verify that the site was part of a slow pathway according to the following criteria: (1) an acceleration of the heart rate to the pacing rate with little change in the configuration of the QRS complexes; (2) a long stimulus to QRS delay; (3) stimulus to QRS delay is identical to the local electrogram to QRS delay; (4) the postpacing return cycle at the pacing site is identical to the cycle length of the ventricular tachycardia. ${ }^{12} 13$

\section{EFFECTIVE REFRACTORY PERIOD}

The effective refractory period was measured at the apex of the right ventricle by the extrastimulus technique before and after the administration of mexiletine. The longest coupling interval which failed to capture the myocardium was defined as the effective refractory period. The effective refractory period was measured in the same way in the area of slow conduction. The basic cycle length was $400 \mathrm{~ms}$.

\section{DRUG ADMINISTRATION}

Mexiletine was given orally for one to two weeks in four patients in a dose of $300 \mathrm{mg} / \mathrm{d}$, and intravenously in five patients at dose of $125-150 \mathrm{mg}$. The blood concentration was not measured and in no cases were other drugs given at the same time.

\section{ANALYSIS OF DATA}

The cycle length of ventricular tachycardia, the block cycle length, and the effective refractory periods were measured and their changes by mexiletine were compared. The relation between the cycle length of ventricular tachycardia and the block cycle length and their changes were analysed.

The effective refractory period was compared between normal myocardium at the pacing site distant from the area of slow conduction and diseased myocardium in the area 

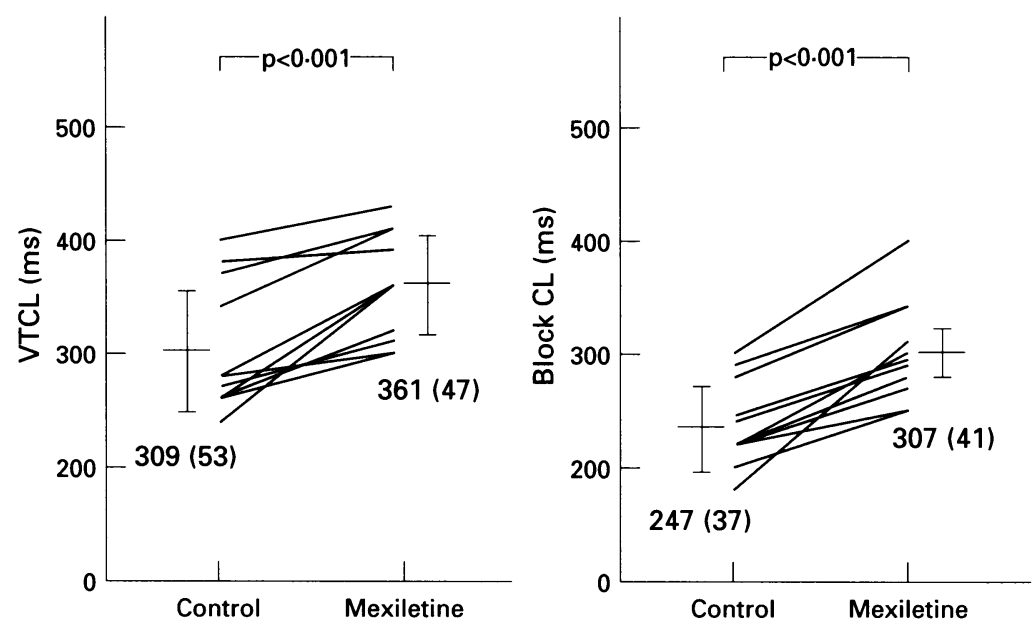

Figure 3 Mexiletine-induced changes of ventricular tachycardia cycle length and block cycle length. After the administration of mexiletine, 11 venricular tachycardias (VTs) with the same morphology were induced in nine patients. VT cycle length and block cycle length were prolonged to a similar degree after mexiletine administration: from 309 (53) to 361 (47) $\mathrm{ms}$ for VT cycle length, and from 247 (37) to 307 (41) ms for block cycle length $(P<0.001)$.

of slow conduction. It was also compared with the block cycle length.

Values are presented as mean (SD). Statistical analysis was performed by $t$ test, and a $P$ value less than 0.05 was considered significant.
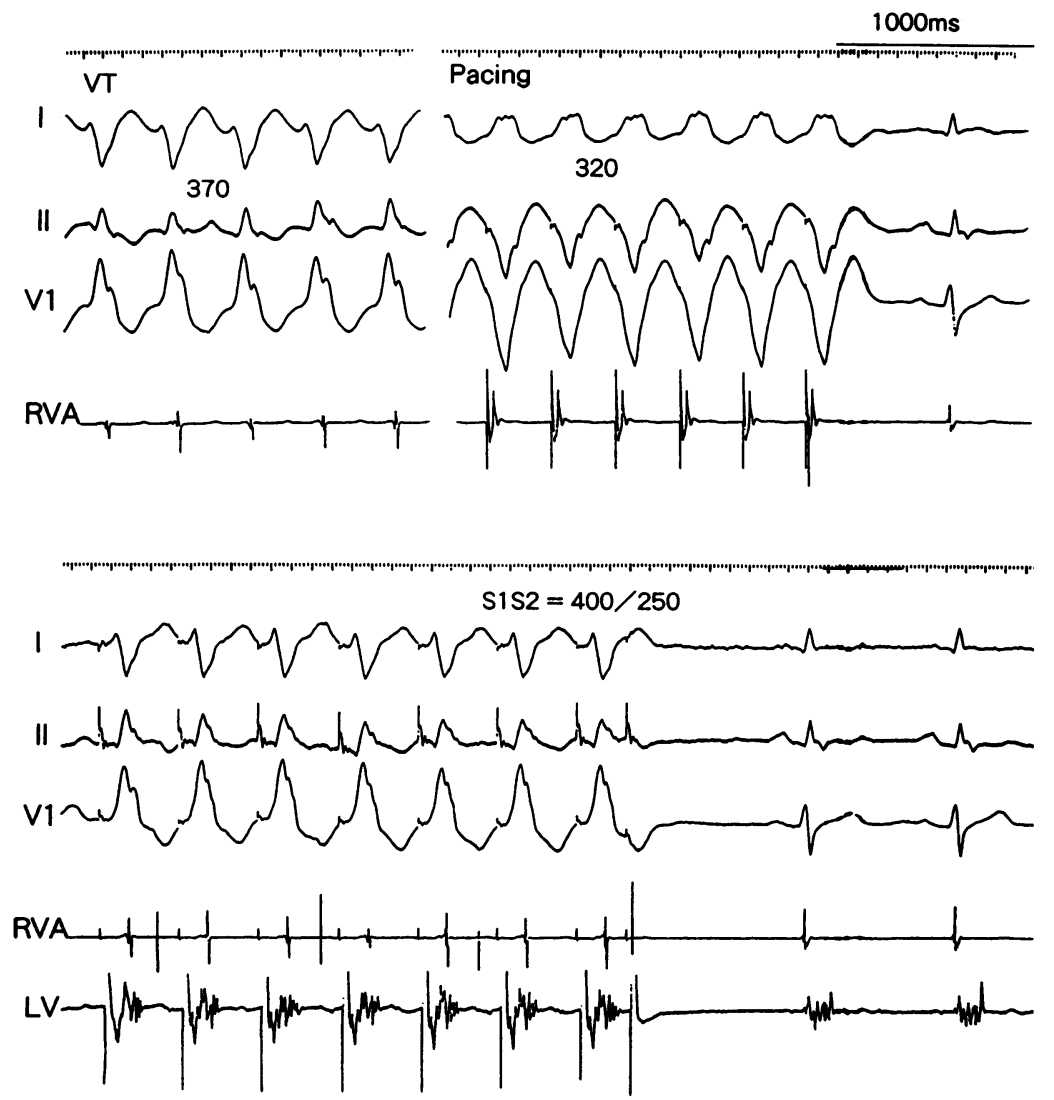

Figure 4 Interruption of ventricular tachycardia (VT) with rapid pacing and effective refractory period in the area of slow conduction. This patient had sustained VT associated with idiopathic VT (case 6 in the table). VT was entrained and interrupted from the right ventricular apex before and after mexiletine administration. The cycle length of VT was $370 \mathrm{~ms}(A)$ and interrupted at $320 \mathrm{~ms}(B)$. However, the effective refractory period measured within the area of slow conduction was $250 \mathrm{~ms}$ at the basic cycle length of 400 $m s$ as shown in C. The stimulus to $Q R S$ interval was $50 \mathrm{~ms}$ and the paced $Q R S$ complex was similar to that of VT.

\section{Results}

INDUCTION AND RAPID PACING OF

VENTRICULAR TACHYCARDIA

Eleven morphologically distinct ventricular tachycardias were induced: there was right bundle branch block morphology in eight and left bundle branch block morphology in three. The mean cycle length of ventricular tachycardia was 309 (53) ms.

All ventricular tachycardias were entrained with rapid pacing: from the right ventricular apex in nine cases, and from the outflow tract in two; they were interrupted at the cycle length of 247 (37) ms. Both constant fusion and progressive fusion were confirmed at longer paced cycle lengths (fig 1).

During rapid pacing at the block cycle length, an abrupt transition of the QRS configuration from a fused complex into a fully paced one was observed in all ventricular tachycardias (figs 1 and 2). At the same time, the local electrogram in the area of slow conduction showed an abrupt change in the morphology and the timing of activation, which became identical to that of the fully paced beats. An abrupt loss of constant fusion and a change of the local electrogram in the area of slow conduction were always followed by interruption of ventricular tachycardia, as was evidenced by the cessation of rapid pacing (figs 1 and 2). There was a good correlation between the ventricular tachycardia cycle length and the block cycle length $(r=0.78$, $P<0.01)$ under control conditions.

\section{EFFECT OF MEXILETINE ON VENTRICULAR TACHYCARDIA}

Ventricular tachycardia induced after the administration of mexiletine was significantly prolonged to 361 (47) $\mathrm{ms}$ and interrupted at the block cycle length of 307 (41) $\mathrm{ms}$, as shown in figs 1 and 2 . The changes in the cycle length of ventricular tachycardia and the block cycle length were 18 (12)\% and 23 (8)\%, respectively, and were significant $(\mathrm{P}<$ $0.001)$. These changes are summarised in fig 3. The cycle length of ventricular tachycardia and the block cycle length were also correlated after administration of mexiletine $(r=0.70$, $P<0.01$ ).

\section{EFFECTIVE REFRACTORY PERIOD}

The effective refractory period measured at the apex of the right ventricle $(n=10)$ was unaffected by mexiletine administration: 241 (21) ms before $v 240$ (22) ms after administration of mexiletine, that is, $1(5) \%$. In the area of slow conduction, the effective refractory period was 262 (9) $\mathrm{ms}$ in five patients and longer than that at the pacing site: 242 (15) ms $(P<0.05)$; it changed little after mexiletine: 252 (9) $\mathrm{ms}(\mathrm{P}>0 \cdot 1)$

\section{COMPARISONS BETWEEN EFFECTIVE} REFRACTORY PERIOD AND BLOCK CYCLE LENGTH

The difference between the block cycle length and the effective refractory period measured at the site of origin of ventricular tachycardia in five patients was not significantly different in 
Figure 5 Comparisons between the effecive refractory period and block cycle length. The block cycle length (block CL) was statistically not different from the effective different from the effective
refractory period $(E R P)$ of the area of slow conduction measured in the control state: 286 (42) v 262 (9) $m s(n=5)$. However, after mexiletine administration, the block cycle length was prolonged by 25 (8)\% and was longer than the effective refractory period $(P<$ 0.02). The asterisk means that the block cycle length was prolonged significantly from the control value after mexiltine administration $(P<0.001)$, but this was not the case for the effective refractory period (left). C, control; Mex, after mexiletine administration.

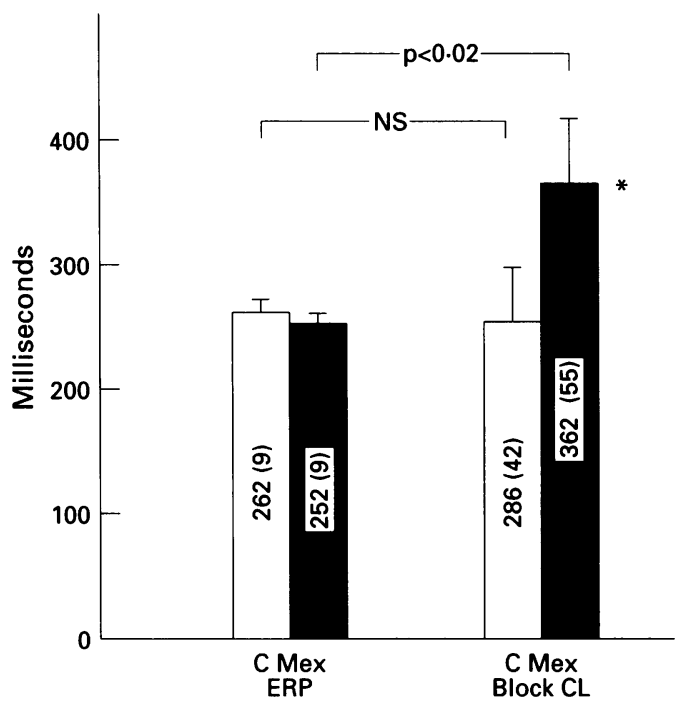

the control state: $286(42) \mathrm{ms} v 262(9) \mathrm{ms}$ $(P>0 \cdot 1)$. After mexiletine administration, the block cycle was, however, prolonged significantly and became longer than the effective period, as shown in figs 4 and 5: 362 (55) $\mathrm{ms} v$ 252 (9) $\mathrm{ms}(\mathrm{P}<0.02)$.

\section{Discussion}

EFFECTS OF MEXILETINE ON VENTRICULAR TACHYCARDIA

Mexiletine is known to block fast sodium channels of cardiac cells and also to shorten the duration of the action potential. ${ }^{8-10}$ As previous studies have shown, the effect of mexiletine on the cycle length of ventricular tachycardia at the therapeutic dosage was significant but smaller than those observed in other class IA or IC drugs. ${ }^{61415}$ Since mexiletine acts preferentially on the depressed fast sodium channel, ${ }^{16-18}$ a smaller effect of the drug on the cycle length of reentrant ventricular tachycardia must be related to the fact that the majority of the myocardium within the area of slow conduction has a normal resting potential ${ }^{19-21}$; the non-significant change in the effective refractory periods was a not unexpected finding for mexiletine.

However, mexiletine resulted in a prolongation of the VT-interrupting critical paced cycle length: the block cycle length which can be considered as the critical paced cycle length at which $1: 1$ orthodromic conduction fails within the area of slow conduction as discussed below.

\section{ENTRAINMENT AND INTERRUPTION OF VENTRICULAR TACHYCARDIA}

From the inclusion criteria, all ventricular tachycardias were entrained and interrupted at a critical cycle length: the block cycle length. ${ }^{126}$ The characteristic findings during pacing at the block cycle length were, firstly, an abrupt loss of constant fusion in the surface electrocardiogram; secondly, a change in the presystolic electrogram, known as localised block by Waldo ${ }^{3}$; and thirdly, there was always an interruption of ventricular tachycardia, as evident after the cessation of rapid pacing (figs 1 and
2). These findings can be considered to represent orthodromic block, which is the cause of the interruption of ventricular tachycardia with rapid pacing at that cycle length.

In the canine model of ventricular tachycardia, it was shown that the stimulated wavefronts from a certain site enter the central common pathway through a narrow corridor without disrupting the reentrant circuit; increasing the rate causes termination of ventricular tachycardia ${ }^{22} 23$ which would represent a pacing induced orthodromic block within the zone of slow conduction.

Though the precise site of conduction block could not be specified in the present study, it would be safe to assume that the block cycle length represents the critical paced cycle length at which 1:1 conduction fails within the area of slow conduction.

DETERMINANTS OF THE BLOCK CYCLE LENGTH As the cycle length of ventricular tachycardia was prolonged, the block cycle length was also prolonged significantly after mexiletine administration (fig 3). The action of mexiletine reflects a combination of the drug induced shortening of the action potential duration and the counteracting prolongation of time dependent refractoriness through unblocking of sodium channels during diastole. ${ }^{16-18}$ The fact that the effective refractory period changed little but the block cycle length did change means that the effective refractory period was not the determinant of the block cycle length.

In the presence of class I antiarrhythmic drugs, the recovery of the maximum upstroke velocity $\left(\mathrm{V}_{\max }\right)$ of the action potential or the sodium inward current may lag behind completion of repolarisation, especially at higher frequencies. ${ }^{16-18}$ Indeed, quinidine and procainamide have long been known to prolong the refractory period well beyond the duration of the action potential when this parameter was measured by the maximum follow frequency, ${ }^{24} 25$ which would be similar to the block cycle length of the present study.

In our previous study, ${ }^{26}$ mexiletine was shown to depress $V_{\max }$ at a frequency higher than $2.0 \mathrm{~Hz}$ and facilitated an induction of block in intercellular conduction by reducing the excitatory currents. The prolongation of the block cycle length would be explained by such action of mexiletine within the area of slow conduction and might be called "postrepolarisation refractoriness".

\section{LIMITATIONS}

The present study has several limitations. First, we studied ventricular tachycardia associated with various types of underlying heart disease. However, all ventricular tachycardias not associated with coronary artery disease have been shown to be equally entrained, 126 and we were studying ventricular tachycardias that shared a common mechanism: reentry.

As in an earlier report ${ }^{3}$ and in our previous studies, ${ }^{126}$ orthodromic block within the area of slow conduction was considered responsible for the interruption of the ventricular tachycardia. At the block cycle length, we assumed 
that $1: 1$ conduction fails, resulting in orthodromic block in the area of slow conduction, but we have to wait for high density mapping to reveal the precise site of block within the reentrant circuit in human ventricular tachycardia, as demonstrated in the canine model. ${ }^{22} 23$

Though the effective refractory period was measured in the area of slow conduction, it cannot be representative of the whole reentrant pathway. However, a markedly prolonged action potential duration has not been reported in the diseased myocardium where ventricular tachycardia originates. ${ }^{19-21}$

The effective refractory period was measured at a basic cycle length of $400 \mathrm{~ms}$, which was longer than the cycle length of ventricular tachycardia: 361 (45) ms. Because of the frequency dependent shortening of the duration of the action potential and the fast kinetics of mexiletine, the effective refractory period should be measured at exactly the same rate.

Since rapid pacing was performed from a site distant from the tachycardia circuit, the conduction of the paced wavefronts might be affected by the intervening normal myocardium between the pacing site and the entrance to the central common pathway; however, the action of mexiletine on normal myocardium has been shown to be smaller than on diseased myocardium. ${ }^{27}$ In ventricular tachycardia of the rabbit, production of an echo wave within the slow conduction zone has been shown to be the cause of interruption, ${ }^{28}$ but so far this remains to be proven in human ventricular tachycardia.

\section{CONCLUSIONS}

Ventricular tachycardia was entrained and interrupted at a critical cycle length (block cycle length) when rapid pacing was repeated at progressively shorter cycle lengths. The abrupt loss of constant fusion and concomitant change of the local electrogram in the area of slow conduction were compatible with orthodromic block within the area of slow conduction, and the block cycle length was considered to be the longest cycle length at which $1: 1$ conduction failed within the reentrant circuit.

Mexiletine prolonged the block cycle length as well as the cycle length of ventricular tachycardia but the effective refractory period changed little. The change in the duration of the action potential must be unrelated to the prolongation of the cycle length of ventricular tachycardia and the block cycle length.

The block cycle length must be determined by factors other than the duration of the action potential, possibly by a depression of intercellular conduction.

1 Aizawa Y, Naitoh N, Kusano Y, Uchiyama H, Washizuka $T$, Shibata A. Frequencyof presumed reentry with an excitable gap in sustained ventricular tachycardia unassociated with coronary artery disease. Am $₹$ Cardiol 1993; 72:916-21.

2 Aizawa Y, Niwano S, Chinushi M, et al. Incidence and the mechanism of interruption of reentrant ventricular tachycardia with rapid ventricular pacing. Circulation 1992: 85:589-95.
3 Waldo AL, Henthorn RW, Plumb VJ, MacLean WAH. Demonstration of the mechanism of transient entrainment and interruption of ventricular tachycardia with ment atrial pacing. $\mathcal{F} \mathrm{Am}$ Coll Cardiol 1984;3:422-30.

4 Okumura K, Henthorn RW, Epstein AE, Plumb VJ, Waldo AL. Further observations on transient entrainment: importance of pacing site and properties of the components of the reentry circuit. Circulation 1985;72 1293-307.

5 Kay GN, Epstein AE, Plumb VJ. Incidence of reentry with an excitable gap in ventricular tachycardia: a prospective evaluation utilizing transient entrainment. $\mathscr{f} \mathrm{Am}$ Coll Cardiol 1988;11:530-8.

6 Aizawa Y, Naitoh N, Takahashi K, et al. Procainamideinduced change in reentrant ventricular tachycardia with special reference to the tachycardia-interrupting critical paced cycle length during transient entrainment with rapid pacing. Ipn Heart $\mathcal{f} 1994 ; 35: 611-23$.

7 Vaughan Williams EM. Classification of antidysrhythmic drugs. In: Sandoe E, Flensted-Jensen E, Olsen HK, eds. drugs. In: Sandoe E, Flensted-Jensen E, Olsen HK, eds.
Cardiac arrhythmias. Sodertalj: AD Astra, 1970:449-72.

8 Carmeliet E, Vereecke J. Electrogenesis of action potential and automaticity. In: Berne RM, Sperelakis N, Geiger and automaticity. In: Berne RM, Sperelakis N, Geiger tem, vol 1 . Bethesda: American Physiological Society, tem, vol 1. Bethe

9 Ebihara E, Johnson EA. Fast sodium current in cardiac muscle. A quantitative description. Biophys $\mathcal{f} 1980 ; 32$ 757-79.

10 Grant AO, Starmer CF, Strauss HC. Antiarrhythmic drug action. Blockade of the inward sodium current. Circ Res 1984;55:427-39.

11 Josephson ME, Horowitz LN, Spielman SR, Greenspan AM. Role of catheter mapping in the preoperative evalua-
tion of ventricular tachycardia. Am $\mathcal{f}$ Cardiol 1982; 49:207-410.

12 Morady F, Frank R, Kay WH, et al. Identification and catheter ablation of a zone of slow conduction in the reentrant circuit of ventricular tachycardia. $¥ \mathrm{Am}$ Coll Cardiol 1988;11:775-82.

13 Fontaine G, Tonet FJ, Grossgeat Y. Identification of a zone of slow conduction appropriate for ventricular tachycardia ablation: theoretical and practical considerations. $P A C E$ 1989;12:262-7.

14 Josephson ME. Recurrent ventricular tachycardia. In: Clinical cardiac electrophysiology. Technique and interpreta tions, 2nd ed. Philadelphia: Lea \& Febiger, 1993. 417-615.

15 Kay GN, Epstein AE, Plumb VJ. Preferential effect of procainamide on the reentrant circuit of ventricular tachycardia. 7 Am Coll Cardiol 1989;14:382-90.

16 Boyden PA, Wit AL. Pharmacology of the antiarrhythmic drugs. In: Rosen MR, Hoffman BF, eds. Cardiac therapy. Boston: Martinus Nijhoff, 1983:171-234.

17 Hondeghem LM, Katzung BG. Antiarrhythmic agents: the modulated receptor mechanism of action of sodium and calcium channel blocking drugs. Annu Rev Pharmacol Toxicol 1984;24:387-423.

18 Campbell TJ. Kinetics of onset of rate-dependent effects of class-I antiarrhythmic drugs are important in determining their effects on refractoriness in guinea-pig ventricle, and provide a theoretical basis for their subclassification. Cardiovasc Res 1983;17:344-52

19 Gilmour RF, Heger J, Prystowsky EN, Zipes DP. Cellular electrophysiologic abnormalities of diseased human ventricular myocardium. $A m$ f Cardiol 1983;51:137-44

20 Spear JF, Horowitz LN, Hodess AB, MacVaugh H, Moore EN. Cellular electrophysiology of human myocardial infarction. Circulation 1979;59:247-56.

21 De Bakker JMT, van Capelle FJL, Janse M, et al. Reentry as a cause of ventricular tachycardia in patients with chronic ischemic heart disease: electrophysiologic and anatomic correlation. Circulation 1988;77:589-606.

22 El-Sherif N, Mehra R, Gough WB, Zeiler RH. Reentrant ventricular arrhythmias in the late myocardial infarction period: interruption of reentrant circuits by cryothermal period: interruption of reentrant circuits

23 Waldecker B, Coromilas J, Saltman AE, Dillon SM, Wit AL. Overdrive stimulation of functional reentrant circuits causing ventricular tachycardia in the infarcted canine heart. Resetting and entrainment. Circulation 1993;87: heart. Reseti

24 Vaughan Williams EM, Szerekes L. A comparison of test for antifibrillatory action. $\mathrm{Br} \mathcal{f}$ Pharmacol 1961;17: 424-32.

25 Szerekes L, Vaughan Williams EM. Antifibrillatory action. $\mathcal{f}$ Physiol (Lond) 1962;160:470-82.

26 Kodama I, Wilde A, Janse MJ, Durrer D, Yamada K Combined effects of hypoxia, hyperkalemia and acidosis on membrane action potential and excitability of guineapig ventricular muscle. $\mathcal{F ~} \mathrm{Mol}$ Cell Cardiol 1984;16: 247-59.

27 Aizawa Y, Chinushi M, Shiba M, Abe A, Shibata A Preferential action of mexiletine on the diseased myocardium [abstr]. In: Symposium on Recent Advances in Studies of Cardiac Ion Channels and Arrhythmias, Tokyo, 1995.

28 Brugada P, Boersma L, Abdollah H, Krichhof C, Allessie M. Echo-wave termination of ventricular tachycardia. A common mechanism of termination of reentrant arrhythmias by various pharmacological interventions. Circulation 1992;85:1879-87. 